\title{
Article \\ Characteristics of Surgical Necrotizing Enterocolitis: Is It Different from Medical Necrotizing Enterocolitis? A Single-Center Retrospective Study
}

\author{
Ara Cho ${ }^{1}$, Dayoung Ko ${ }^{1}$, JoongKee Youn ${ }^{1}$, Hee-Beom Yang ${ }^{2}$ and Hyun-Young Kim ${ }^{1, *}$ \\ 1 Department of Surgery, Division of Pediatric Surgery, Seoul National University Hospital, 101 Daehakro, \\ Chongno-gu, Seoul 03080, Korea; ara501616@gmail.com (A.C.); kodayoung@gmail.com (D.K.); \\ jkyoun@gmail.com (J.Y.) \\ 2 Department of Surgery, Division of Pediatric Surgery, Seoul National University Bundang Hospital, 82, \\ Gumi-ro 173 Beon-gil, Bundang-gu, Seongnam-si 13620, Korea; eeulere@naver.com \\ * Correspondence: spkhy02@snu.ac.kr; Tel.: +82-2-2072-2478
}

check for updates

Citation: Cho, A.; Ko, D.; Youn, J.; Yang, H.-B.; Kim, H.-Y. Characteristics of Surgical Necrotizing Enterocolitis: Is It Different from Medical Necrotizing Enterocolitis? A Single-Center Retrospective Study. Children 2021, 8, 1148. https:// doi.org/10.3390/children8121148

Academic Editor:

Jörn-Hendrik Weitkamp

Received: 21 September 2021

Accepted: 4 December 2021

Published: 6 December 2021

Publisher's Note: MDPI stays neutral with regard to jurisdictional claims in published maps and institutional affiliations.

Copyright: (c) 2021 by the authors. Licensee MDPI, Basel, Switzerland. This article is an open access article distributed under the terms and conditions of the Creative Commons Attribution (CC BY) license (https:/ / creativecommons.org/licenses/by/ $4.0 /)$.

\begin{abstract}
Background: Necrotizing enterocolitis (NEC) is one of the leading causes of death in newborns despite improvements in the care of critically ill neonates. Approximately 50-70\% of the cases are managed by medical therapy. However, the remaining patients require surgical intervention. The purpose of our study was to analyze the factors associated with patients requiring surgical treatment compared to patients requiring only medical treatment; (2) Method: Patients diagnosed with necrotizing enterocolitis over a period of 14 years (January 2003-December 2016) in a single tertiary referral children's hospital were retrospectively enrolled. Demographics and clinical data were collected through the medical record and were analyzed using Pearson's $\chi^{2}$ test, t-tests, and linear regression; (3) Results: A total of 189 NEC patients were analyzed. In the surgical NEC group, gestational age was lower $(p=0.018)$, body weight at birth was lower $(p=0.034)$, comorbidity with respiratory distress syndrome (RDS) was higher $(p=0.005)$, the days of antibiotic use were greater $(p=0.014)$, the percentage of breast milk feeding was lower $(p=0.001)$, and the length of hospital stay was longer $(p<0.000)$. The in-hospital mortality between the two groups was not significantly different $(p=0.196)$. In multivariate logistic analysis, breast milk feeding remained less associated with surgical NEC (OR $=0.366,95 \%$ CI: 0.164-0.817), whereas the length of hospital stay was more associated with surgical NEC (OR = 1.010, 95\% CI: 1.001-1.019); (4) Conclusion: Comparing medical and surgical NEC, a significantly lower percentage of surgical NEC patients were fed breast milk and their hospital stays were longer.
\end{abstract}

Keywords: NEC; surgical; medical; risk factor; outcome; neonate

\section{Introduction}

NEC is characterized by intestinal necrosis in the ileum, jejunum, and colon and is one of the major causes of morbidity and mortality in premature infants [1]. Occurring in 0.5 to 5 per 1000 live births, NEC is more common in low-weight infants [2,3]. The pathophysiology of NEC is not fully understood, but several risk factors have been reported. Immaturity, ischemia, altered gut microflora, decreased mucin barrier, increased gut permeability, decreased gut immunity, and certain types of enteral feeding formulas have been reported as risk factors for NEC [4-7]. Several of the identified risk factors result in oxidative stress, inflammation, and ultimately necrosis of the neonatal bowel $[4,5]$.

NEC is diagnosed by a combination of clinical symptoms and signs, including feeding intolerance, abdominal distension, and bloody stools. Radiologic features include pneumatosis intestinalis, portal venous gas, and pneumoperitoneum [8].

When NEC is diagnosed or strongly suspected, medical management begins. Medical management should stop all enteral feeding, perform gastric decompression via orogastric tubes, 
inject intravenous fluids, inject intravenous broad-spectrum antibiotics, and ensure central intravenous access [9]. Surgical intervention, which consists of removing necrotic intestine and performing an ileostomy or anastomosis, is required in 30-50\% of all NEC cases [10].

There is still insufficient information on whether the clinical characteristics of surgical and medical NEC patients differ. Thus, the purpose of this study was to compare medical and surgical NEC patients to identify clinical factors that identify which NEC patients will require surgical intervention.

\section{Materials and Methods}

\subsection{Study Design}

This study was a retrospective study whose protocol was approved by the institutional review board of the Seoul National University Hospital (SNUH) (IRB number: 1911-0061074). The informed consent was waived because of the retrospective design of the study. We retrospectively evaluated patients with NEC over a period of 14 years (March 2003-September 2016) at the Seoul National University Children's Hospital (SNUCH). The diagnosis of NEC was done in reference to the modified Bell staging [11]. This study enrolled stage I, II, and III of enterocolitis. Stage I was suspected and unproven enterocolitis, most of these children did not progress to stage II or III. Stage II was definite enterocolitis which included pneumatosis intestinalis, ascites, and tenderness of the abdomen. Patients in stage I and II were medically treated. Stage III was enterocolitis with signs of peritonitis and intestinal perforation. Patients in stage III were treated surgically. Patients diagnosed with NEC who did not receive surgical therapy were defined as medical NEC patients, and patients who received surgical treatment were defined as surgical NEC patients.

\subsection{Variables}

Data on the factors related to patient demographics, maternal factors, clinical factors, laboratory, and treatment factors were recorded. The demographics included sex, gestational age (GA) (weeks), body weight at birth (g), body weight at birth (extremely low body weight [ELBW] less than $1000 \mathrm{~g}$, very low body weight [VLBW] between $1000 \mathrm{~g}$ and $1500 \mathrm{~g}$ ), location of the birth (hospital in-born or out-born), the method of delivery (vaginal delivery or cesarean section), small size for gestational age, multiple births, Apgar score at one minute and five minutes, cord $\mathrm{pH}$, body temperature at neonatal intensive care unit (NICU) admission, comorbidities (respiratory distress syndrome (RDS), moderate to severe bronchopulmonary dysplasia (BPD), retinopathy of prematurity (ROP) surgery, intraventricular hemorrhage (IVH) grade $>3$, periventricular leukomalacia (PVL), medical patent ductus arteriosus (PDA), and surgical patent ductus arteriosus), associated gastrointestinal (GI) anomalies, and associated cardiac anomalies (except PDA, patent foramen ovale (PFO), or arterial switch operation (ASO)). Maternal factors included: maternal age (years), maternal diabetes mellitus (DM), hypertension (HTN), history of maternal steroid usage, polyhydramnios, oligohydramnios, placental abruption, cause of prematurity (spontaneous labor, premature rupture of membrane (PROM), fetal distress, and other causes), and the number of days of antibiotic use for PROM. Laboratory and treatment factors included: antibiotic use, transfusions, exchange transfusions, indomethacin or ibuprofen use, postnatal steroid use, sepsis, number of days of antibiotic use (days), highest WBC count, lowest hemoglobin level, lowest platelet count, highest C-reactive protein (CRP) level, day of first feed, day first full feeding accomplished, type of material feed (breast milk or other formula), time of meconium pass (hours), length of hospital stay (days), and in-hospital mortality. All patients were followed-up until hospital discharge or death.

\subsection{Statistical Analysis}

To evaluate categorical variables, we used $\chi^{2}$ tests or Fisher's exact test. To compare continuous variables, we used Student's t-test or the Mann-Whitney U-test. Factors associated with surgical NEC were identified by univariate analysis and their corresponding $p$-values. Variables with $p$-values $<0.05$ were included in the multivariate analysis. Based 
on the logistic regression model, correlations between the identified factors and surgical NEC were analyzed. Statistical analysis was performed using the Statistical Package for Social Science (SPSS) version 25.0.

\section{Results}

\subsection{Patients Demographics}

Of the 189 patients, 102 were treated with medical intervention and 98 patients were treated with surgical intervention. The two groups are compared in Table 1 . The proportion of male patients was $57.5 \%$ in the surgical NEC group and $31.7 \%$ in the medical NEC group. The average gestational age was $26.5 \pm 2.5$ weeks in the surgical NEC group and $27.4 \pm 2.4$ weeks in the medical NEC group $(p=0.018)$. The average body weight at birth was $821.5 \pm 260.2 \mathrm{~g}$ in the surgical NEC patients and $888.5 \pm 227.3 \mathrm{~g}$ in the medical NEC patients $(p=0.034)$. The percentage of patients who were small for gestational age was $36.1 \%$ in the surgical NEC group and $26.7 \%$ in the medical NEC group. RDS is a disorder primarily caused by the deficiency of pulmonary surfactant in immature lung. RDS was diagnosed based on a clinical manifestation of respiratory failure and characteristic radiologic findings. RDS was seen in $82.6 \%$ of the surgical NEC patients and $64.3 \%$ of the medical NEC patients $(p=0.005)$.

Table 1. Patient demographics.

\begin{tabular}{|c|c|c|c|c|}
\hline Characteristics & $\begin{array}{c}\text { Total } \\
(\mathrm{N}=189)\end{array}$ & $\begin{array}{l}\text { Surgical NEC } \\
\quad(\mathbf{N}=87)\end{array}$ & $\begin{array}{l}\text { Medical NEC } \\
\quad(\mathrm{N}=102)\end{array}$ & $p$-Value \\
\hline Male & $110(58.2)$ & $50(57.5)$ & $60(31.7)$ & 0.851 \\
\hline Gestational age (weeks) & $27.0 \pm 2.5$ & $26.5 \pm 2.5$ & $27.4 \pm 2.4$ & 0.018 \\
\hline Body weight at birth (g) & $857.7 \pm 244.6$ & $821.5 \pm 260.2$ & $888.5 \pm 227.3$ & $0.034 *$ \\
\hline \multicolumn{5}{|l|}{ Body weight } \\
\hline ELBW (<1.0 kg) & $131(69.3)$ & $62(71.3)$ & $69(67.6)$ & \multirow{2}{*}{0.591} \\
\hline VLBW $(<1.5 \mathrm{~kg})$ & $58(30.7)$ & $25(28.7)$ & $33(32.4)$ & \\
\hline \multicolumn{5}{|l|}{ Location of birth } \\
\hline In-born & $117(61.6)$ & $48(55.2)$ & $69(67.6)$ & \multirow[b]{2}{*}{0.078} \\
\hline Out-born & $72(38.1)$ & $39(44.8)$ & $33(32.4)$ & \\
\hline \multicolumn{5}{|l|}{ Method of delivery } \\
\hline Vaginal delivery & $64(34.8)$ & $30(34.9)$ & $34(34.7)$ & \multirow{2}{*}{0.978} \\
\hline Cesarean section & $120(65.2)$ & $56(65.1)$ & $64(65.3)$ & \\
\hline Small for gestational age & $49(31.0)$ & $26(36.1)$ & $23(26.7)$ & 0.205 \\
\hline Multiple births & $90(49.7)$ & $43(52.4)$ & $47(47.5)$ & 0.506 \\
\hline \multicolumn{5}{|l|}{ Apgar score } \\
\hline Apgar score at $1 \mathrm{~min}$ & $3.5 \pm 2.1$ & $3.4 \pm 2.1$ & $3.5 \pm 2.2$ & 0.784 \\
\hline Apgar score at $5 \mathrm{~min}$ & $5.7 \pm 2.0$ & $5.6 \pm 2.0$ & $5.8 \pm 1.9$ & 0.514 \\
\hline Cord $\mathrm{pH}$ & $7.3 \pm 0.1$ & $7.3 \pm 1.0$ & $7.3 \pm 1.0$ & 0.373 \\
\hline $\begin{array}{c}\text { Body temperature } \\
\text { at NICU admission }\left({ }^{\circ} \mathrm{C}\right)\end{array}$ & $35.8 \pm 1.1$ & $35.8 \pm 1.3$ & $35.7 \pm 1.0$ & $0.506^{*}$ \\
\hline \multicolumn{5}{|l|}{ Comorbidity } \\
\hline RDS & $134(72.8)$ & $71(82.6)$ & $63(64.3)$ & 0.005 \\
\hline Moderate to severe BPD & $86(63.7)$ & $43(71.7)$ & $43(57.3)$ & 0.085 \\
\hline ROP operation & $52(48.6)$ & $30(57.7)$ & $22(40.0)$ & 0.067 \\
\hline IVH grade $>3$ & $23(15.4)$ & $10(15.2)$ & $13(15.7)$ & 0.932 \\
\hline PVL & $18(10.2)$ & $9(10.8)$ & $9(9.7)$ & 0.799 \\
\hline \multicolumn{5}{|l|}{ PDA } \\
\hline Medical PDA & $89(49.2)$ & $43(51.2)$ & $46(47.4)$ & \multirow{2}{*}{$0.473^{* * *}$} \\
\hline Surgical PDA & $52(28.7)$ & $25(29.8)$ & $27(27.8)$ & \\
\hline Associated GI anomaly & $2(1.1)$ & $1(1.1)$ & $1(1.0)$ & $1.000^{* *}$ \\
\hline $\begin{array}{l}\text { Associated cardiac anomaly } \\
\text { (except PDA, PFO, ASO) }\end{array}$ & $12(6.5)$ & $6(7.0)$ & $6(6.1)$ & 0.801 \\
\hline
\end{tabular}

Data are presented as mean $\pm \mathrm{SD}$, or number (\%). $p$-value: comparison of surgical and surgical NEC. * Mann-Whitney U test. ${ }^{* *}$ Fisher's exact test. ${ }^{* * *}$ Linear by linear association. NEC $=$ necrotizing enterocolitis, NICU $=$ neonatal intensive care unit, RDS = respiratory distress syndrome, $\mathrm{BPD}=$ bronchopulmonary dysplasia, $\mathrm{ROP}=$ retinopathy of prematurity, $\mathrm{IVH}=$ intraventricular hemorrhage, $\mathrm{PVL}=$ periventricular leukomalacia, $\mathrm{PDA}=$ patent ductus arteriosus, $\mathrm{GI}=$ gastrointestinal, $\mathrm{PFO}=$ permanent foramen ovale, $\mathrm{ASO}=$ arterial switch operation . 


\subsection{Maternal Factors, Laboratory Factors and Treatment Factors of Patients}

Maternal factors of the NEC patients are listed in Table 2. Laboratory and treatment factors are shown in Table 3. More surgical NEC patients were treated with antibiotics $(94.0 \%)$ than medical NEC patients (85.9\%). The number of antibiotic use days was $30.4 \pm 27.4$ in the surgical NEC patients and $20.9 \pm 21.9$ in the medical NEC patients $(p=0.014)$. The highest WBC count was $21.9 \pm 14.2 \times 103 / \mu \mathrm{L}$ in the surgical NEC patients and $21.1 \pm 13.0 \times 103 / \mu \mathrm{L}$ in the medical NEC patients. The highest CRP level was $11.5 \pm 48.6 \mathrm{mg} / \mathrm{dL}$ in the surgical NEC patients and $4.5 \pm 5.1 \mathrm{mg} / \mathrm{dL}$ in the medical NEC patients. Exclusive breast feeding occurred in $29.5 \%$ of the surgical NEC patients, while the rest were either fed formula or a mixture of the two (70.5\%). This is in contrast to medical NEC patients, where $57.6 \%$ were exclusively breast fed, and $42.4 \%$ were fed formula or a mixture of the two $(p=0.001)$. The length of hospital stay was $99.7 \pm 61.5$ days for the surgical NEC patients and $73.5 \pm 37.4$ in the medical NEC patients $(p=0.000)$. The in-hospital mortality rate was $16.1 \%$ in the surgical NEC patients and $9.8 \%$ in the medical NEC patients.

Table 2. Maternal factors.

\begin{tabular}{ccccc}
\hline Characteristics & $\begin{array}{c}\text { Total } \\
(\mathbf{N}=\mathbf{1 8 9})\end{array}$ & $\begin{array}{c}\text { Surgical NEC } \\
(\mathbf{N}=\mathbf{8 7})\end{array}$ & $\begin{array}{c}\text { Medical NEC } \\
(\mathbf{N}=\mathbf{1 0 2})\end{array}$ & $p$-Value \\
\hline Maternal age (years) & $32.8 \pm 4.4$ & $32.9 \pm 5.0$ & $32.6 \pm 4.0$ & 0.642 \\
Maternal DM & $8(5.1)$ & $4(5.4)$ & $4(4.8)$ & $1.000 * *$ \\
Maternal HTN & $24(14.8)$ & $8(10.8)$ & $16(18.2)$ & 0.188 \\
Maternal steroid history & $79(54.9)$ & $39(58.2)$ & $40(51.9)$ & 0.451 \\
Polyhydramnios & $8(6.6)$ & $5(9.4)$ & $3(4.4)$ & $0.296^{* *}$ \\
Oligohydramnios & $20(16.5)$ & $9(17.0)$ & $11(16.2)$ & 0.906 \\
Placental abruption & $5(3.2)$ & $1(1.4)$ & $4(4.8)$ & $0.372 * *$ \\
Cause of prematurity & & & & \\
Preterm labor with spontaneous labor & $39(24.7)$ & $18(24.3)$ & $21(25.0)$ & $33(39.3)$ \\
Preterm labor with PROM & $63(39.3)$ & $30(40.5)$ & $18(21.4)$ & $0.650 * * *$ \\
Othetal distress & $28(17.7)$ & $10(13.5)$ & $12(14.3)$ & $9.1 \pm 14.9$ \\
Antibiotic use for PROM (days) & $28(17.7)$ & $16(21.3)$ & 0.301
\end{tabular}

Data are presented as mean $\pm \mathrm{SD}$, or number (\%). $p$-value: comparison of surgical and surgical NEC. ${ }^{* *}$ Fisher's exact test. ${ }^{* * *}$ Linear by linear association. $\mathrm{DM}=$ diabetes mellitus, $\mathrm{HTN}=$ hypertension, $\mathrm{PROM}=$ premature rupture of membrane.

Table 3. Laboratory and treatment factors.

\begin{tabular}{ccccc}
\hline Characteristics & $\begin{array}{c}\text { Total } \\
(\mathbf{N}=\mathbf{1 8 9})\end{array}$ & $\begin{array}{c}\text { Surgical NEC } \\
\mathbf{( N = 8 7 )}\end{array}$ & $\begin{array}{c}\text { Medical NEC } \\
(\mathbf{N}=\mathbf{1 0 2})\end{array}$ & $p$-Value \\
\hline Antibiotics & $158(89.8)$ & $79(94.0)$ & $79(85.9)$ & 0.074 \\
Transfusion & $150(84.7)$ & $75(90.4)$ & $75(79.8)$ & 0.051 \\
Exchange transfusion & $1(0.6)$ & $1(1.2)$ & $0(0.0)$ & $0.468^{* *}$ \\
Indomethacin or ibuprofen & $104(60.1)$ & $46(57.5)$ & $58(62.4)$ & 0.515 \\
Postnatal steroids & $12(7.2)$ & $6(7.9)$ & $6(6.6)$ & 0.746 \\
Sepsis & $92(52.3)$ & $50(59.5)$ & $42(45.7)$ & 0.066 \\
Antibiotic usage (days) & $25.5 \pm 25.1$ & $30.4 \pm 27.4$ & $20.9 \pm 21.9$ & $0.014 *$ \\
Highest WBC (x/ $\mu$ L) & $21.5 \pm 13.5$ & $21.9 \pm 14.2$ & $21.1 \pm 13.0$ & 0.676 \\
Lowest Hb (g/dL) & $8.7 \pm 2.0$ & $8.9 \pm 2.3$ & $8.5 \pm 1.6$ & $0.231 *$ \\
Lowest Plt (x/ $\mu \mathrm{L})$ & $111.0 \pm 110.1$ & $130.7 \pm 146.1$ & $93.7 \pm 62.8$ & $0.213 *$ \\
Highest CRP (mg/dL) & $7.6 \pm 32.7$ & $11.5 \pm 48.6$ & $4.5 \pm 5.1$ & $0.962 *$ \\
First diet (days) & $7.8 \pm 8.6$ & $7.0 \pm 6.4$ & $8.2 \pm 9.8$ & 0.423 \\
Full feeding (days) & $34.3 \pm 28.3$ & $42.7 \pm 38.1$ & $29.4 \pm 18.9$ & $0.069 *$ \\
Feeding material & & & & 0.001 \\
Breast milk & $71(46.4)$ & $18(29.5)$ & $53(57.6)$ & 0.001 \\
Formula \& mixed & $82(53.6)$ & $43(70.5)$ & $39(42.4)$ & 0.878 \\
Meconium passing time (hours) & $14.9 \pm 16.9$ & $15.2 \pm 22.0$ & $14.7 \pm 12.2$ & $0.000 *$
\end{tabular}

Data are presented as mean $\pm \mathrm{SD}$, or number (\%). $p$-value: comparison of surgical and surgical NEC. ${ }^{*}$ Mann-Whitney

$\mathrm{U}$ test. ${ }^{* *}$ Fisher's exact test. $\mathrm{WBC}=$ white blood cell, $\mathrm{Hb}=$ hemoglobin, $\mathrm{Plt}=$ platelet, $\mathrm{CRP}=\mathrm{C}$-reactive protein . 


\subsection{Risk Factors of Surgical NEC}

In a multivariate analysis of the factors associated with surgical NEC, six factors with $p$-values $<0.05$ were analyzed (Table 4). Surgical NEC patients had reduced odds of being exclusively breast fed than medical NEC patients (OR 0.366; 95\% CI: 0.164-0.817). Surgical NEC patients also had increased odds of a longer hospital stay compared to medical NEC patients (OR 1.010; 95\% CI: 1.001-1.019).

Table 4. Multivariate logistic analysis of the factors associated with surgical necrotizing enterocolitis.

\begin{tabular}{ccc}
\hline & $\begin{array}{c}\text { Adjusted Odds Ratio } \\
\mathbf{( 9 5 \%} \text { Confidence Interval) }\end{array}$ & $p$-Value \\
\hline Breast milk feeding & $0.366(0.164-0.817)$ & 0.014 \\
RDS & $2.784(0.981-7.905)$ & 0.054 \\
Length of hospital stay (days) & $1.010(1.001-1.019)$ & 0.036 \\
\hline
\end{tabular}

RDS = respiratory distress syndrome.

\section{Discussion}

Early predictors of progression to surgical NEC are of great interest in NEC research [12]. In the absence of a clear pneumoperitoneum, signs in physical examinations, such as a fixed abdominal mass and abdominal wall erythema, are the best evidence of intestinal necrosis [13]. According to Kosloske's retrospective cohort study of 147 NEC patients, pneumoperitoneum, portal venous gas, and positive paracentesis were the best indicators of surgical intervention, with a positive predictive value of $100 \%$ and a prevalence of $410 \%$. Positive paracentesis refers to positive bacteria in brown fluid or gram stains. However, there is controversy over the indications for paracentesis. Kosloske recommends paracentesis in cases of clinical deterioration despite medical management or with extensive pneumatosis intestinalis [14]. Kosloske's recommendation of portal venous gas as a predictor of surgical intervention has been questioned by other retrospective reviews that have reported a low value for portal venous gas in predicting NEC. Sharma et al. reported that about half of the NEC patients with portal venous gas survived without surgery [14-16].

Tepas et al. reported a predictive model including seven metabolic derangements. Seven parameters (positive blood culture, acidosis, bandemia, thrombocytopenia, hyponatremia, hypotension, and neutropenia) were scored in a binary manner to assess disease severity and determine the need for surgical intervention [17]. Using this model, outcomes were improved in patients with Bell's stage II or III NEC compared to using presence of pneumoperitoneum in decision-making [18].

Biomarker studies have been conducted to predict which patients will progress to surgical NEC. Severe thrombocytopenia $(<100 \times 109 / \mathrm{L})$ correlated with indications of disease extension, mortality, and laparotomy [19-21]. Reisinger et al. reported that urinary serum amyloid A (SAA) levels and platelet counts could be used to predict surgical NEC accurately, with sensitivities of $94 \%$ and specificities of $83 \%$ [22]. Fecal calprotectin (S100A8/S100A9 heterodimer) distinguishes between Bell's stage II and Bell's stage III with $76 \%$ sensitivity and $92 \%$ specificity. Another fecal protein, S100A12, was higher in the bowel perforation group of patients with suspected NEC than in the other group [23]. Some studies have shown that elevated CRP implies an advanced stage of NEC, [24] while other studies have reported no association with disease severity. Significantly higher CRP levels were reported in surgical NEC patients compared to medical NEC patients $(2625 \mathrm{pg} / \mathrm{mL}$ vs. $156 \mathrm{pg} / \mathrm{mL}$, respectively, $p<0.001$ ) [25].

Miriam et al. compared 170 patients in Italy and found that surgical NEC patients had lower GA $(p<0.0001)$, lower birth weight $(p=0.0006)$, and lower onset body weight $(p<0.0001)$ compared to medical NEC patients. There were also more ELBW patients $(p=0.0033)$, more PDA $(p=0.0033)$, more use of NSAIDs $(p=0.0229)$, earlier onset of NEC $(p=0.0003)$, more days to reach full enteral feeding $(p<0.0001)$, longer hospital stays $(p<0.0001)$, higher mortality $(p=0.0003)$, and higher platelet counts $(p=0.0071)$ in the 
surgical NEC patients. In multivariate regression analysis, later onset of NEC, higher $\mathrm{pH}$ values, and intestinal pneumatosis on abdominal $\mathrm{x}$-ray were associated with a lower risk of surgery, whereas higher CRP levels at NEC onset were associated with a higher risk of surgery [26].

Similar to the results of the Miriam study, we found that surgical NEC patients had a lower GA and lower birth weight and longer hospital stay, but we did not find significant correlations with the proportion of ELBW patients, PDA, days to reach full feeding, and hospital mortality. Regarding laboratory factors, we did not find that WBC, hemoglobin levels, platelet counts, or CRP levels were associated with surgical NEC. Further prospective studies are required to collect information on new factors and to corroborate those factors found to be important in previous studies.

A strength of this study was that we identified formula and mixed feeding as a significant risk factor for surgical NEC. A previous study of patients in the early postnatal period reported that the components of breast milk were protective against NEC, not only in exclusive feeding but also in partial feeding, with a dose-dependent relationship between exclusively of breast milk feeding and its protective effect [26]. Breast milk contains both nutrient and non-nutrient components. The non-nutrient components include immunoglobulins and lactoferrin, which aid in intestinal adaptation, maturation, food tolerance, and protection against infective and inflammatory disorders [27]. The absence of protective proteins in breast milk and the presence of foreign bovine proteins can be detrimental to intestinal immune balance. It can also drive microbiota dysbiosis, which is a disturbance in the composition of the microbiota in the intestine. The impact of probiotics and breast milk in NEC patients indicates that intestinal dysbiosis is an important mechanism in the progression of NEC [28]. It is recommended that in preterm babies with known risk factors of NEC, it would be helpful to have human milk feeding in advance; because it has protective effect. In addition to the importance of breast milk, they reported on enteral feeding strategies and microbiota analysis. Slower and intermediate rates of progression of enteral feeding strategies were associated with a higher risk of NEC. Also, less favorable and intermediate direct-breastfeeding policies were associated with higher risk of NEC. Microbiota analysis resulted in the association between Clostridium neonatale and Staphylococcus aureus with NEC. This report implies that feeding strategies and microbiota as well as feeding materials are important and should be considered in future studies [29].

There were some limitations to this study. First, there are no definite unit protocols on trophic feeding in NEC patients. However, the doctor determines the feeding schedule by comprehensively considering the physical exam, radiologic findings, and laboratory results. In case of suspected peritonitis or perforation, trophic feeding is delayed. Elsewhere, early trophic feeding is recommended for nutrition and growth.

This study is retrospective in nature and data was collected retrospectively. Some detailed data were not collected, such as the timing of antibiotic usage and the presence of chorioamnionitis in the mother. Also, there is a lack of data on physical examinations and radiologic findings associated with surgical treatment decisions. Identifying significant factors by using all available evidence including imaging, clinical data, laboratory findings, and maternal factors can help improve the determination of the best parameters to predict surgical intervention.

\section{Conclusions}

In conclusion, we found that lower levels of exclusive breast milk feeding and longer hospital stays were significantly associated with the need for surgery in NEC patients. Further multi-center studies with larger sample sizes and long-term follow-up will clarify the factors associated with the progression from medical NEC to surgical NEC.

Author Contributions: Conceptualization, H.-Y.K.; Methodology, J.Y. and D.K.; Software, A.C.; Validation, H.-B.Y. and H.-Y.K.; Formal Analysis, A.C.; Investigation, A.C.; Resources, J.Y.; Data Curation, A.C. and D.K.; Writing-Original Draft Preparation, A.C.; Writing-Review \& Editing, 
D.K. and H.-Y.K.; Visualization, A.C.; Supervision, H.-Y.K.; Project Administration, H.-Y.K.; Funding Acquisition, None. All authors have read and agreed to the published version of the manuscript.

Funding: This research received no external funding.

Institutional Review Board Statement: The study was conducted according to the guidelines of the Declaration of Helsinki, and approved by the Institutional Review Board of SEOUL NATIONAL UNIVERSITY HOSPITAL (1911-006-1074).

Informed Consent Statement: Patient consent was waived due to retrospective feature of this study.

Conflicts of Interest: The authors declare no conflict of interest.

\section{References}

1. Marseglia, L.; D'Angelo, G.; Manti, S.; Aversa, S.; Reiter, R.J.; Antonuccio, P.; Centorrino, A.; Romeo, C.; Impellizzeri, P.; Gitto, E. Oxidative Stress-Mediated Damage in Newborns with Necrotizing Enterocolitis: A Possible Role of Melatonin. Am. J. Perinatol. 2015, 32, 905-909.

2. Lin, P.W.; Stoll, B.J. Necrotising enterocolitis. Lancet 2006, 368, 1271-1283. [CrossRef]

3. Guthrie, S.O.; Gordon, P.V.; Thomas, V.; Thorp, J.A.; Peabody, J.; Clark, R.H. Necrotizing enterocolitis among neonates in the United States. J. Perinatol. 2003, 23, 278-285. [CrossRef]

4. Morgan, J.A.; Young, L.; McGuire, W. Pathogenesis and prevention of necrotizing enterocolitis. Curr. Opin. Infect. Dis. 2011, 24, 183-189. [CrossRef]

5. Marseglia, L.; D’Angelo, G.; Manti, M.; Aversa, S.; Fiamingo, C.; Arrigo, T.; Barberi, I.; Mami, C.; Gitto, E. Visfatin: New marker of oxidative stress in preterm newborns. Int. J. Immunopathol. Pharmacol. 2016, 29, 23-29. [CrossRef]

6. Torrazza, R.M.; Neu, J. The altered gut microbiome and necrotizing enterocolitis. Clin. Perinatol. 2013, 40, 93-108. [CrossRef] [PubMed]

7. Martin, R.; Nauta, A.J.; Ben Amor, K.; Knippels, L.M.; Knol, J.; Garssen, J. Early life: Gut microbiota and immune development in infancy. Benef. Microbes 2010, 1, 367-382. [CrossRef]

8. Eaton, S.; Rees, C.M.; Hall, N.J. Current research in necrotizing enterocolitis. Early Hum. Dev. 2016, 97, 33-39. [CrossRef]

9. Rich, B.S.; Dolgin, S.E. Necrotizing Enterocolitis. Pediatr. Rev. 2017, 38, 552-559. [CrossRef]

10. Cristofalo, E.A.; Schanler, R.J.; Blanco, C.L.; Sullivan, S.; Trawoeger, R.; Kiechl-Kohlendorfer, U.; Dudell, G.; Rechtman, D.J.; Lee, M.L.; Lucas, A.; et al. Randomized trial of exclusive human milk versus preterm formula diets in extremely premature infants. J. Pediatr. 2013, 163, 1592-1595. [CrossRef]

11. Neu, J. Necrotizing enterocolitis: The search for a unifying pathogenic theory leading to prevention. Pediatr Clin. N. Am. 1996, 43, 409-432. [CrossRef]

12. Grave, G.D.; Nelson, S.A.; Walker, W.A.; Moss, R.L.; Dvorak, B.; Hamilton, F.A.; Higgins, R.; Raju, T.N. New therapies and preventive approaches for necrotizing enterocolitis: Report of a research planning workshop. Pediatr. Res. 2007, 62, 510-514. [CrossRef]

13. Kosloske, A.M. Indications for operation in necrotizing enterocolitis revisited. J. Pediatr. Surg. 1994, 29, 663-666. [CrossRef]

14. Sharma, R.; Tepas, J.J., 3rd; Hudak, M.L.; Wludyka, P.S.; Mollitt, D.L.; Garrison, R.D.; Bradshaw, J.A.; Sharma, M. Portal venous gas and surgical outcome of neonatal necrotizing enterocolitis. J. Pediatr. Surg. 2005, 40, 371-376. [CrossRef] [PubMed]

15. Molik, K.A.; West, K.W.; Rescorla, F.J.; Scherer, L.R.; Engum, S.A.; Grosfeld, J.L. Portal venous air: The poor prognosis persists. J. Pediatr. Surg. 2001, 36, 1143-1145. [CrossRef] [PubMed]

16. Cikrit, D.; Mastandrea, J.; West, K.W.; Schreiner, R.L.; Grosfeld, J.L. Necrotizing enterocolitis: Factors affecting mortality in 101 surgical cases. Surgery 1984, 96, 648-655. [PubMed]

17. Tepas, J.J., 3rd; Sharma, R.; Leaphart, C.L.; Celso, B.G.; Pieper, P.; Esquivia-Lee, V. Timing of surgical intervention in necrotizing enterocolitis can be determined by trajectory of metabolic derangement. J. Pediatr. Surg. 2010, 45, 310-313. [PubMed]

18. Tepas, J.J., 3rd; Leaphart, C.L.; Plumley, D.; Sharma, R.; Celso, B.G.; Pieper, P.; Quilty, J.; Esquivia-Lee, V. Trajectory of metabolic derangement in infants with necrotizing enterocolitis should drive timing and technique of surgical intervention. J. Am. Coll. Surg. 2010, 210, 847-852. [CrossRef] [PubMed]

19. Kenton, A.B.; O’Donovan, D.; Cass, D.L.; Helmrath, M.A.; Smith, E.O.; Fernandes, C.J.; Washburn, K.; Weihe, E.K.; Brandt, M.L. Severe thrombocytopenia predicts outcome in neonates with necrotizing enterocolitis. J. Perinatol. 2005, 25, 14-20. [CrossRef]

20. Ververidis, M.; Kiely, E.M.; Spitz, L.; Drake, D.P.; Eaton, S.; Pierro, A. The clinical significance of thrombocytopenia in neonates with necrotizing enterocolitis. J. Pediatr. Surg. 2001, 36, 799-803. [CrossRef] [PubMed]

21. Miner, C.A.; Fullmer, S.; Eggett, D.L.; Christensen, R.D. Factors affecting the severity of necrotizing enterocolitis. J. Matern. Fetal Neonatal Med. 2013, 26, 1715-1719. [CrossRef] [PubMed]

22. Reisinger, K.W.; Kramer, B.W.; Van der Zee, D.C.; Brouwers, H.A.; Buurman, W.A.; van Heurn, E.; Derikx, J.P. Non-invasive serum amyloid A (SAA) measurement and plasma platelets for accurate prediction of surgical intervention in severe necrotizing enterocolitis (NEC). PLoS ONE 2014, 9, e90834. [CrossRef] [PubMed]

23. Dabritz, J.; Jenke, A.; Wirth, S.; Foell, D. Fecal phagocyte-specific S100A12 for diagnosing necrotizing enterocolitis. J. Pediatr. 2012, 161, 1059-1064. [CrossRef] [PubMed] 
24. Cetinkaya, M.; Ozkan, H.; Koksal, N.; Akaci, O.; Ozgur, T. Comparison of the efficacy of serum amyloid A, C-reactive protein, and procalcitonin in the diagnosis and follow-up of necrotizing enterocolitis in premature infants. J. Pediatr. Surg. 2011, 46, 1482-1489. [CrossRef]

25. Benkoe, T.; Reck, C.; Gleiss, A.; Kettner, S.; Repa, A.; Horcher, E.; Rebhandl, W. Interleukin 8 correlates with intestinal involvement in surgically treated infants with necrotizing enterocolitis. J. Pediatr. Surg. 2012, 47, 1548-1554. [CrossRef] [PubMed]

26. Furman, L.; Taylor, G.; Minich, N.; Hack, M. The effect of maternal milk on neonatal morbidity of very low-birth-weight infants. Arch. Pediatr. Adolesc. Med. 2003, 157, 66-71. [CrossRef] [PubMed]

27. Quigley, M.; Embleton, N.D.; McGuire, W. Formula versus donor breast milk for feeding preterm or low birth weight infants. Cochrane Database Syst. Rev. 2019, 7, CD002971. [CrossRef] [PubMed]

28. Underwood, M.A. Probiotics and Human Milk Oligosaccharides in Premature Infants. Neoreviews 2019, 20, e1-e11. [CrossRef] [PubMed]

29. Rozé, J.C.; Ancel, P.Y.; Lepage, P.; Martin-Marchand, L.; Al Nabhani, Z.; Delannoy, J.; Picaud, J.C.; Lapillonne, A.; Aires, J.; Durox, M.; et al. Nutritional strategies and gut microbiota composition as risk factors for necrotizing enterocolitis in very-preterm infants. Am. J. Clin. Nutr. 2017, 106, 821-830. [CrossRef] [PubMed] 